Claremont Colleges

Scholarship@ Claremont

All HMC Faculty Publications and Research

HMC Faculty Scholarship

9-24-2004

\title{
Improved Phase Modulation for an En-face Scanning Three-dimensional Optical Coherence Microscope
}

Barbara M. Hoeling

California State Polytechnic University - Pomona

Mary E. Peter

Daniel C. Petersen

Harvey Mudd College

Richard C. Haskell

Harvey Mudd College

\section{Recommended Citation}

Hoeling, Barbara M., Mary E. Peter, Daniel C. Petersen, and Richard C. Haskell. "Improved phase modulation for an en-face scanning three-dimensional optical coherence microscope." Review of Scientific Instruments 75.10 (2004): 3348-3350. DOI: 10.1063/

1.1790555

This Article is brought to you for free and open access by the HMC Faculty Scholarship at Scholarship @ Claremont. It has been accepted for inclusion in All HMC Faculty Publications and Research by an authorized administrator of Scholarship @ Claremont. For more information, please contact scholarship@cuc.claremont.edu. 


\title{
Improved phase modulation for an en-face scanning three-dimensional optical coherence microscope
}

\author{
Barbara M. Hoeling, Mary E. Peter, Daniel C. Petersen, and Richard C. Haskell ${ }^{\mathrm{a}}$ \\ Physics Department, Harvey Mudd College, 241 East Twelfth Street, Claremont, California 91711
}

(Received 18 December 2003; accepted 15 July 2004; published 24 September 2004)

\begin{abstract}
We have previously described an inexpensive method for modulating the interferometer of an en-face scanning, focus-tracking, three-dimensional optical coherence microscope (OCM). In this OCM design, a reference mirror is mounted on a piezoelectric stack driven at a resonance frequency of about $100 \mathrm{kHz}$. We perform a partial discrete Fourier transform of the digitally sampled output fringe signal. In the original design, we obtained the amplitude of the backscattered light by summing the powers in the fundamental $(1 \omega)$ and first harmonic $(2 \omega)$ of the modulation frequency. We used the particular piezoamplitude that eliminates the effects of interferometer phase drift. However, as the reference mirror was stepped to scan at different sample depths, variations in the back-coupled reference power added noise to the fringe signal at the fundamental piezodriving frequency. We report here a technique to eliminate the effects of this piezowobble by using instead the sum of the $2 \omega$ and $3 \omega$ powers as a measure of the backscattered light intensity. Images acquired before and after this improvement are presented to illustrate the enhancement to image quality deep within the sample. (C) 2004 American Institute of Physics. [DOI: 10.1063/1.1790555]
\end{abstract}

\section{INTRODUCTION}

Optical coherence tomography/microscopy (OCT/OCM) is an imaging technique with micrometer-scale resolution that can measure the optical properties of biological tissues. ${ }^{1,2}$ It is typically based on a Michelson interferometer with the sample in one arm and a reference mirror in the other. A broadband light source provides a coherence gate that ensures that the observed interference fringes arise from scatterers located at a well-defined depth within the sample.

Most OCT systems produce two-dimensional crosssectional images of the sample by translating the sample or beam in one lateral direction while quickly and repeatedly scanning the optical path length of the reference arm. Different depths of the sample come into equal path length position with the reference mirror during such a depth scan, allowing the determination of fringe amplitude and phase for each volume element (voxel) along the optical beam.

In contrast, our fiber-based optical coherence microscope is designed to take three-dimensional (3D) images by performing a series of en-face scans at different sample depths. ${ }^{3}$ Galvoscanners move the beam laterally in an $x-y$ plane normal to the beam axis, then the sample head (galvoscanners, fiber end, and focusing lens) is stepped down for the next $x-y$ plane scan while the position of the reference mirror is adjusted to keep the beam waist coincident with the equal path length position ("focus tracking"). The lateral resolution is thus given by the size of the beam waist of $5 \mu \mathrm{m}$ and is preserved throughout the whole 3D scan. Fringes are produced by oscillating the reference mirror with a small amplitude. As we described earlier, ${ }^{4}$ our design for such a phase modulation involves attaching the reference mirror to a pi-

${ }^{a)}$ Electronic mail: richard_haskell@hmc.edu ezoelectric stack driven at resonance. We were able to achieve a modulation frequency of about $125 \mathrm{kHz}$, facilitating high-speed data acquisition, and we used a displacement amplitude of only $350 \mathrm{~nm}$ in order to preserve good depth resolution. Since this modulation amplitude of about one fringe is very small, slow phase drifts due to thermal expansion and contraction of the optical fibers can significantly degrade the output signal. In our original design, we circumvented this problem by choosing a certain amplitude of mirror oscillation for which the sum of the powers in the fundamental $(\omega)$ and first-harmonic $(2 \omega)$ piezofrequency is independent of the phase drift. ${ }^{3,5,6}$

However, the output fringe signal at the fundamental pi-

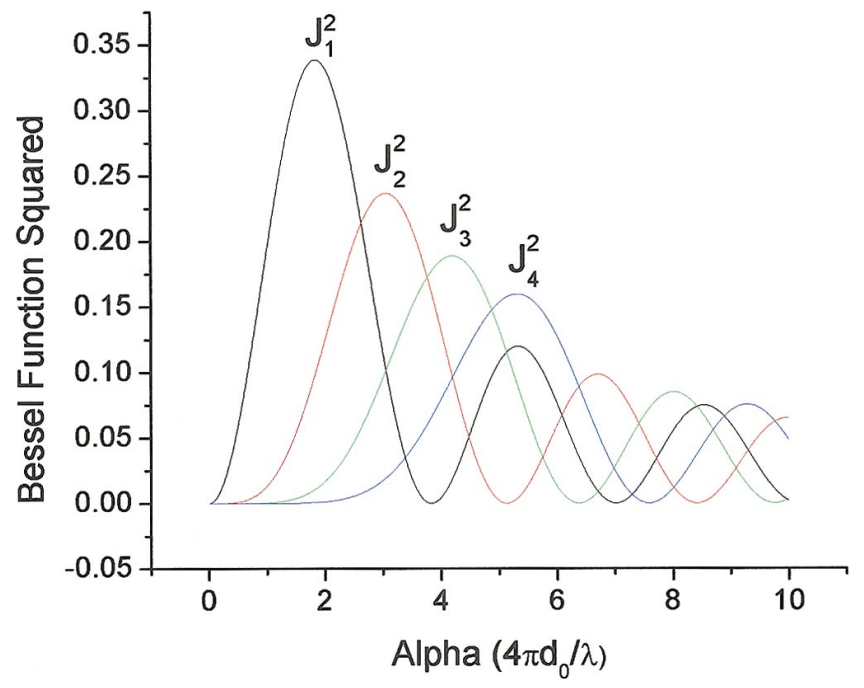

FIG. 1. (Color) Squares of the Bessel functions as a function of the dimensionless piezoamplitude $\alpha=4 \pi d_{0} / \lambda$. At $\alpha=2.63, J_{1}^{2}=J_{2}^{2}$, while at $\alpha=3.77$, $J_{2}^{2}=J_{3}^{2}$. 
ezofrequency included a small oscillation due to variations in the back-coupled reference power caused by a very slight rotation of the piezostack as it expanded and contracted. While this oscillation could be greatly reduced by careful piezoalignment for one particular position of the reference mirror, it became significant when the piezo was moved to image a deeper plane within the sample, and effectively limited imaging to depths of less than $1 \mathrm{~mm}$. Since we are now interested in imaging very weakly scattering samples-like corneal tissue that in principle allow penetration depths of several millimeters-this limitation must be overcome. We will describe in detail below how we eliminated the effect of this piezowobble by using the sum of the powers in the $2 \omega$ and $3 \omega$ harmonic frequencies as our signal, while at the same time maintaining insensitivity to thermal phase drifts. A comparison of images taken with the old and new signal processing methods clearly shows the reduction in noise achieved with the new scheme.

\section{THEORY}

With a sinusoidal voltage applied to the piezo, the optical power output of the OCM interferometer can be written as

$$
P_{\text {out }}=P_{\text {ref }}+P_{\text {sample }}+2 \sqrt{P_{\text {ref }} P_{\text {sample }}} \cos [\alpha \sin \omega t+\phi],
$$

where $P_{\text {ref }}$ is the optical power in the reference beam, and $P_{\text {sample }}$ is the backscattered power in the sample beam. The dimensionless piezoamplitude is $\alpha=4 \pi d_{0} / \lambda$, where the peak-to-peak amplitude of the piezo-oscillation is $2 d_{0}$, and the wavelength of the light in vacuum is $\lambda$. The frequency of the piezo-oscillation is $\omega$. The first part of the phase $(\alpha \sin \omega t)$ of the interference term varies rapidly $(\omega / 2 \pi$ $=56.85 \mathrm{kHz})$ due to the oscillation of the piezomirror. The second phase term, $\phi$, is a slow drift on a time scale of a few seconds to a few minutes arising from a variety of factors, such as the temperature variation of the optical fibers in the interferometer.

The ac-coupled output of the OCM photodetector is due to the interference term in Eq. (1):

$$
V_{\text {interference }}(t)=V_{0} \cos [\alpha \sin \omega t+\phi],
$$

where $V_{0} \propto 2 \sqrt{P_{\text {ref }} P_{\text {sample }}}$ is the amplitude of the fringe signal. It can be shown that the ac power in this interference signal is

$$
\text { ac power in } \begin{aligned}
V_{\text {interference }}= & 2 V_{0}^{2}\left[J_{1}^{2}(\alpha)+J_{3}^{2}(\alpha)+J_{5}^{2}(\alpha)\right. \\
& +\ldots] \sin ^{2} \phi+2 V_{0}^{2}\left[J_{2}^{2}(\alpha)\right. \\
& \left.+J_{4}^{2}(\alpha)+J_{6}^{2}(\alpha)+\ldots\right] \cos ^{2} \phi
\end{aligned}
$$

where the $J_{n}^{2}(\alpha)$ are the squares of the Bessel functions, and $2 V_{0}^{2} J_{n}^{2}(\alpha) \sin ^{2} \phi$ (or $2 V_{0}^{2} J_{n}^{2}(\alpha) \cos ^{2} \phi$ ) is the power in the $n$th harmonic frequency if $n$ is odd (or even). The fundamental frequency of the piezo-oscillation corresponds to $n=1$.

Figure 1 shows the squares of the Bessel functions $J_{1}$ through $J_{4}$ as a function of the dimensionless piezoamplitude $\alpha$. It can be seen from this graph that for the special value of

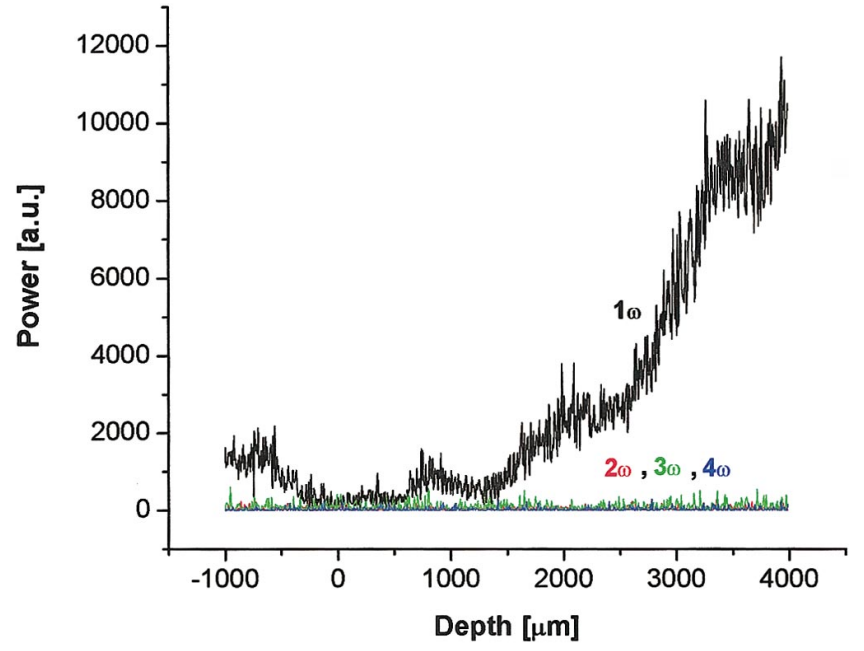

FIG. 2. (Color) Piezowobble noise measured without a sample in each of the first four-harmonic frequencies as a function of scanning depth. The piezostack was driven at $56.85 \mathrm{kHz}$, the lowest resonance frequency of the aluminum disk on which it is mounted, and the dimensionless piezoamplitude was $\alpha=3.77$.

$\alpha=2.63\left(2 d_{0}=0.418 \lambda\right)$, the Bessel functions $J_{1}^{2}(\alpha)$ and $J_{2}^{2}(\alpha)$ have the same value. The sum of the powers in $\omega$ and $2 \omega$ is, therefore, independent of the slow phase drift $\phi$ for this particular piezoamplitude. ${ }^{3,5,6}$ We perform a partial discrete Fourier transform on our fringe signal and initially chose to operate our OCM at $\alpha=2.63$ while using the square root of the sum of the powers in $\omega$ and $2 \omega$ as the amplitude of the fringes for each volume element (voxel). This measure of the fringe amplitude was indeed insensitive to the phase drift, though it left us with a signal strength of only $0.214 \times 2 V_{0}^{2}$, about $60 \%$ of what could theoretically be achieved by summing the powers in all harmonics.

\section{MEASUREMENT OF THE PIEZOWOBBLE}

As the piezostack expands and contracts, the reference mirror glued to its surface does not translate perfectly along the optical axis, but rotates slightly during the motion. Consequently, the optical power reflected back into the reference arm fiber $\left[P_{\text {ref }}\right.$ in Eq. (1)] can vary periodically, thereby contributing to the ac-coupled output of the OCM photodetector. Careful alignment of the reference mirror in the cat's eye retroreflector can reduce this problem only for one particular mirror position, but as the reference mirror is translated to image different depths in the sample, the piezowobble oscillations become substantial. Figure 2 shows a graph of the power in the first four-harmonic frequencies of the fringe signal taken without a sample as a function of scanning depth. It can be seen clearly that the piezowobble is contained entirely in the fundamental piezofrequency and increases very rapidly beyond a depth of $1 \mathrm{~mm}$. In contrast, the noise power in the higher harmonics is negligible and independent of the scanning depth.

It is important to understand why the effects of piezowobble appear only at the fundamental frequency $\omega$. The piezo stack responds linearly to the stimulating voltage applied at its resonance frequency, so both mirror displacement and tilt are sinusoidal functions of time at this same fre- 


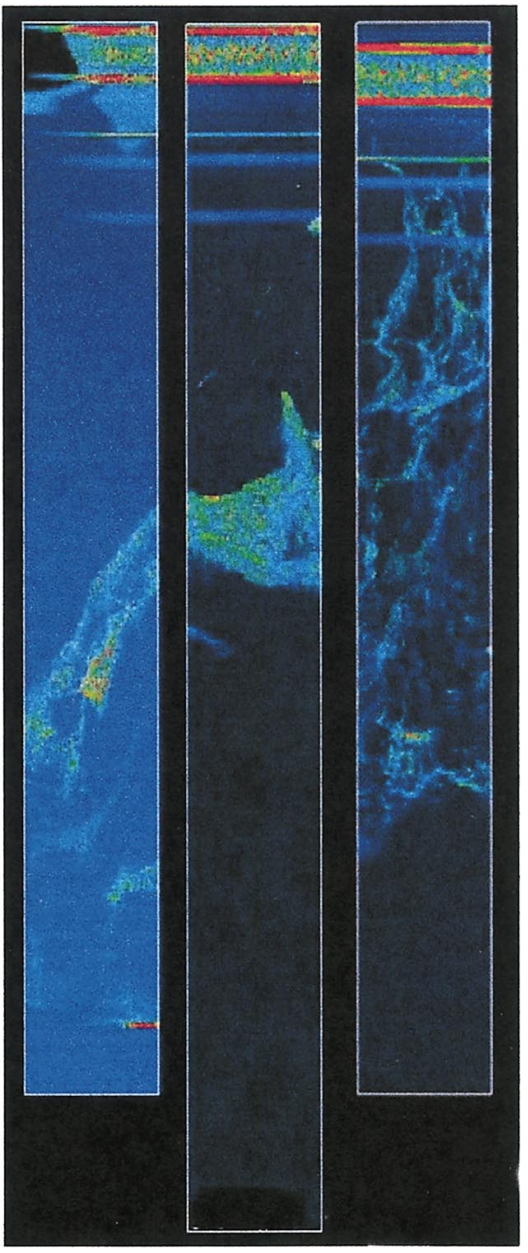

FIG. 3. (Color) Three volume-rendered OCM images of collagen sponges. The left image $(0.5 \mathrm{~mm} \times 0.5 \mathrm{~mm} \times 4.0 \mathrm{~mm}$ deep $)$ was created by using the sum of the powers in $1 \omega$ and $2 \omega$. The center image $(0.5 \mathrm{~mm} \times 0.5 \mathrm{~mm}$ $\times 4.5 \mathrm{~mm}$ deep $)$ and the right image $(0.5 \mathrm{~mm} \times 0.5 \mathrm{~mm} \times 4.0 \mathrm{~mm}$ deep $)$ were created by using the sum of the powers in $2 \omega$ and $3 \omega$.

quency $\omega$. The fringe signal in Eq. (2) is a nonlinear function of the mirror displacement, so the power in the fringe signal is distributed over many harmonics. In contrast, the small mirror tilt gives rise to a very small sinusoidal modulation of the reference beam power that is coupled back into the reference arm fiber. The reference beam power can be written as $P_{\text {ref }}(t)=P_{\text {ave }}+\delta P \sin (\omega t)$ where $\delta P / P_{\text {ave }}$ is on the order of $10^{-4}$ or $10^{-5}$. Substituting this expression for $P_{\text {ref }}(t)$ into the first term in Eq. (1), it can be seen that the small reference power modulation can be the same order of magnitude as the small fringe signal, especially deep into a sample where the fringe signal is very weak. Given the smallness of the mirror tilt, it is not surprising that the reference power modulation depends linearly on the mirror tilt, and therefore occurs at the same frequency $\omega$ as the piezostimulating voltage. Note that according to Eq. (2) the effect of the small modulation of $P_{\text {ref }}(t)$ on the fringe amplitude $V_{0}$ is negligible.

In order to avoid the piezowobble oscillations in the fun- damental frequency of our ac-coupled photodetector output, we choose to use the sum of the powers in $2 \omega$ and $3 \omega$ instead of those in $\omega$ and $2 \omega$ as the measure for the backscattered light from each voxel. Inspection of Fig. 1 shows that in order to achieve phase insensitivity for this case, we now have to operate the piezo with an amplitude of $\alpha=3.77$ $\left(2 d_{0}=0.600 \lambda\right)$. This reduces the signal strength further by about $20 \%$ from $0.214 \times 2 V_{0}^{2}$ to $0.173 \times 2 V_{0}^{2}$, but the effects of piezowobble are eliminated, and the fringe signal is cleaner, particularly deep in the sample.

Figure 3 shows a comparison of three images of collagen sponges, which we use as scaffoldings for corneal tissue cultures. These sponges are very weakly scattering and thus should allow imaging depths of several millimeters. The color map is the same for all three images and makes use of the visible color spectrum, with red signifying the highest scattering amplitude, blue the lowest, and black zerobackscattered light. In the image on the left, the voxel amplitudes were calculated as the square root of the sum of the powers in $\omega$ and $2 \omega$. The increase of the piezowobble noise with depth is evident as the "background" color changes from darker to lighter blue, making it more difficult to distinguish features at greater depths. In contrast, the images in the center and on the right were created using the square root of the sum of the powers in $2 \omega$ and $3 \omega$, and no piezowobble oscillations are present, as can be seen by the evenly dark "background" color. The image in the center demonstrates this noise reduction throughout its whole imaging depth of $4.5 \mathrm{~mm}$, while the image on the right displays the improvement in contrast for the features of the sponge throughout the entire $3 \mathrm{~mm}$ thickness of the sponge.

In conclusion, we have shown that eliminating the effects of piezowobble greatly reduces the noise in our images, especially at greater image depths. By operating the piezo at the special phase insensitive amplitude and by using the square root of the sum of the powers in $2 \omega$ and $3 \omega$ as our fringe amplitude, we have been able to improve the quality of our 3D images considerably.

\section{ACKNOWLEDGMENT}

We gratefully acknowledge support for this work from the National Science Foundation Grant No. DBI-0137973.

${ }^{1}$ Handbook of Optical Coherence Tomography, edited by B. E. Bouma and G. J. Tearney (Marcel Dekker, New York, 2002).

${ }^{2}$ D. Huang, E. A. Swanson, C. P. Lin, J. S. Schuman, W. G. Stinson, W. Chang, M. R. Hee, T. Flotte, K. Gregory, C. A. Puliafito, and J. G. Fujimoto, Science 254, 1178 (1991).

${ }^{3}$ B. M. Hoeling, A. D. Fernandez, R. C. Haskell, E. Huang, W. R. Meyers, D. C. Petersen, S. E. Ungersma, R. Wang, and M. E. Williams, Opt. Express 6, 136 (2000); (http://www.opticsexpress.org/oearchive/source/ 19250.htm).

${ }^{4}$ B. M. Hoeling, A. D. Fernandez, R. C. Haskell, and D. C. Petersen, Rev. Sci. Instrum. 72, 1630 (2001).

${ }^{5}$ S. R. Chinn and E. A. Swanson, Electron. Lett. 29, 2025 (1993).

${ }^{6}$ J. A. Izatt, M. D. Kulkarni, H.-W. Wang, K. Kobayashi, and M. V. Sivak, Jr., IEEE J. Sel. Top. Quantum Electron. 2, 1017 (1996). 